\title{
Global Attractor for the Convective Cahn-Hilliard Equation
}

by

\author{
Xiaopeng ZHAO and Changchun LIU
}

Presented by Bogdan BOJARSKI

Summary. This paper is concerned with the convective Cahn-Hilliard equation. We use a classical theorem on existence of a global attractor to derive that the convective Cahn-Hilliard equation possesses a global attractor on some subset of $H^{2}$.

1. Introduction. We consider the existence of a global attractor on some subset of $\mathrm{H}^{2}$ for the following convective Cahn-Hilliard equation:

$$
\frac{\partial u}{\partial t}+\gamma \Delta^{2} u=\Delta A(u)+\beta \cdot \nabla B(u), \quad x \in \Omega,
$$

where $\Omega$ is a bounded domain in $\mathbb{R}^{n}(n \leq 2), A(u)=\gamma_{2} u^{3}+\gamma_{1} u^{2}-u$, $B(u)=-\frac{1}{4} u^{4}+\frac{1}{2} u^{2}, \gamma>0, \gamma_{2}>0$ and $\gamma_{1}$ are constants.

We assume that $\Omega=\left[0, L_{1}\right] \times\left[0, L_{2}\right], L_{1}, L_{2}>0$. On the basis of physical considerations, as usual 1.1 is supplemented with the periodic boundary condition

$$
\left.\varphi\right|_{x_{i}=0}=\left.\varphi\right|_{x_{i}=L_{i}}, \quad i=1,2,
$$

for $u$ and the derivatives of $u$ at least of order $\leq 3$, and with the initial value condition

$$
u(x, 0)=u_{0}(x) .
$$

Equation (1.1) arises naturally as a continuous model for the formation of facets and corners in crystal growth (see [11, 5]). Here $u(x, t)$ denotes the slope of the interface. The convective term $\beta \cdot \nabla\left(-\frac{1}{4} u^{4}+\frac{1}{2} u^{2}\right)$ (see [5]) stems from the effect of kinetics that provides an independent flux of the order 
parameter, similar to the effect of an external field in spinodal decomposition of a driven system.

Recently there has been renewed interest in the convective Cahn-Hilliard equation. It was K. H. Kwek [6] who first studied equation (1.1) for a special case with a special convection, namely, $B(u)=u$. Based on the discontinuous Galerkin finite element method, he proved the existence of a classical solution. In 2005, C. Liu [8] considered (1.1) with the first boundary condition in one space dimension. Some results on the existence of classical solutions and asymptotic behavior of solutions are established in his paper. Eden and Kalantarov [3] considered the following convective Cahn-Hilliard equation with periodic boundary conditions in one space dimension:

$$
u_{t}-u \cdot \nabla u+\Delta\left(u-u^{3}+\Delta u\right)=0 .
$$

They established the existence of a compact attractor and a finite-dimensional inertial manifold that contains it. Moreover, the Gevrey regularity of solutions on the attractor was established and used to prove that four nodes are determining for each solution on the attractor. Moreover, in [4] equation (1.4) with periodic boundary conditions in 3D was considered by Eden and Kalantarov; they considered the corresponding continuous dynamical system on $\dot{L}^{2}(\Omega)$, and proved that for $(1.4)$ there exist absorbing balls in $\dot{L}^{2}(\Omega)$, $\dot{H}_{\text {per }}^{1}(\Omega)$ and $\dot{H}_{\text {per }}^{2}(\Omega)$. Combining this with the compactness property of the solution semigroup they deduced the existence of a global attractor for 1.4 in $\dot{L}^{2}(\Omega)$. There is an extensive literature concerning the convective Cahn-Hilliard equation; for more recent results we refer the reader to [9, 13] and the references therein.

In this paper, we are interested in a global attractor of the problem 1.1 - 1.3 in $H^{2}$. The paper is organized as follows. In Section 2, we give some preparations and state the main result about the existence of the global attractor. In Section 3, we derive some estimates for the solutions and prove our main result. Some ideas important for our paper come from [2, 7, 12], etc.

2. Preliminaries. We point out one basic fact about (1.1)-1.3): the spatial average of any solution $u$ is preserved, excluding the existence of an absorbing set in $H^{2}(\Omega)$. Indeed, we find

$$
\begin{gathered}
\frac{\partial}{\partial t} \int_{\Omega} u(x, t) d x=0, \\
\int_{\Omega} u(x, t) d x=\int_{\Omega} u_{0} d x, \quad \forall t>0 .
\end{gathered}
$$

We assume that the initial function satisfies $\int_{\Omega} u_{0}(x) d x \leq \kappa$; then it follows that $\int_{\Omega} u(x, t) d x \leq \kappa$ for $t>0$. 
For convenience, in this section we introduce a lemma concerning global existence and uniqueness of the solution of problem 1.1 1.3.

Lemma 2.1. Suppose that $u_{0} \in H^{2}(\Omega)$, Then problem 1.1)-1.3 admits a unique solution $u$ such that

$$
u \in L^{2}\left([0, T] ; H^{4}(\Omega)\right) \cap L^{\infty}\left([0, T] ; H^{2}(\Omega)\right), \quad \forall T>0 .
$$

We can obtain Lemma 2.1 by the standard Galerkin method as in 10 , 1, 17. Since the proof is easy, we omit it.

By Lemma 2.1, we can define the operator semigroup $\{S(t)\}_{t \geq 0}$ in $H^{2}$ by

$$
S(t) u_{0}=u(t), \quad \forall u_{0} \in H^{2}, t \geq 0,
$$

where $u(t)$ is the solution of $(1.1)-(1.3)$ corresponding to the initial value $u_{0}$.

To study the existence of a global attractor, we have to find a complete metric space and prove that there exists a global attractor in that complete metric space. Notice that it is sufficient to consider the global existence and uniqueness of the solution of problem (1.1)- 1.3 for any given initial datum, as described in the previous lemma. However, because $H^{2}(\Omega)$ is not a proper phase space for our problem, we have to choose a complete metric space to work with. On the other hand, since the total mass is conserved for all time, it is not possible for us to have a global attractor in that metric space without any constraints. Instead, we let

$$
\mathscr{U}_{\kappa}=\left\{u\left|u \in H^{2}(\Omega),\right| \int_{\Omega} u d x \mid \leq \kappa\right\},
$$

where $\kappa>0$ is a constant. It is sufficient to see that the restriction of $\{S(t)\}$ to $\mathscr{U}_{\kappa}$ is a well defined semigroup.

Now we can give the main result of this paper.

TheOREM 2.1. Suppose that $\Omega$ is an open bounded domain in $\mathbb{R}^{n}$ $(n \leq 2)$. Then for every $\kappa$ chosen as above, the semiflow associated with the solution $u$ of problem (1.1)-(1.3) possesses in $\mathscr{U}_{\kappa}$ a global attractor $\mathscr{A}_{\kappa}$ which attracts all the bounded sets in $\mathscr{U}_{\kappa}$.

Throughout this paper we denote the $L^{2}, L^{p}$ and $H^{k}(k=1,2,3)$ norms in $\Omega$ simply by $\|\cdot\|,\|\cdot\|_{p}$ and $\|\cdot\|_{H^{k}}$.

3. Proof of Theorem 2.1. In order to prove Theorem 2.1, we establish some a priori estimates for the solution $u$ of problem (1.1)-1.3). In this section we always assume that $\{S(t)\}_{t \geq 0}$ is the semigroup generated by the weak solutions of (1.1) with initial data $u_{0} \in H^{2}(\Omega)$. We have the following lemma.

LEMma 3.1. There exists a bounded set $\mathscr{B}$, whose size depends only on $\kappa$ and $\Omega$, in $\mathscr{U}_{\kappa}$ such that for all the orbits starting from any bounded set 
$B$ in $\mathscr{U}_{\kappa}$, there exists $t_{0}=t_{0}(B) \geq 0$ such that for all $t \geq t_{0}$ all the orbits enter $\mathscr{B}$ and remain there.

Proof. It suffices to prove that there is a positive constant $C$ such that

$$
\|u(t)\|_{H^{2}} \leq C, \quad t \geq t_{0}(B) .
$$

We prove the lemma in the following steps.

STEP 1: $L^{2}$ norm estimate. Multiplying 1.1 with $u$ and integrating over $\Omega$, we obtain

$$
\frac{1}{2} \frac{d}{d t}\|u\|^{2}+\gamma\|\Delta u\|^{2}=\int_{\Omega} u \Delta A(u) d x+\beta \cdot \int_{\Omega} u \nabla B(u) d x .
$$

Hence

$$
\frac{1}{2} \frac{d}{d t}\|u\|^{2}+\gamma\|\Delta u\|^{2}=-\int_{\Omega} A^{\prime}(u)|\nabla u|^{2} d x-\beta \cdot \int_{\Omega} B(u) \nabla u d x .
$$

Since $\gamma_{2}>0$, a simple calculation shows that

$$
A^{\prime}(u)=3 \gamma_{2} u^{2}+2 \gamma_{1} u-1 \geq-C_{0},
$$

where $C_{0}>0$. We also have

$$
\int_{\Omega}\left(\frac{1}{4} u^{4}-\frac{1}{2} u^{2}\right) \nabla u d x=0 .
$$

So it follows from $(3.2)$ that

$$
\frac{1}{2} \frac{d}{d t}\|u\|^{2}+\gamma\|\Delta u\|^{2} \leq C_{0}\|\nabla u\|^{2} \leq C_{0}\|u\| \cdot\|\Delta u\| \leq \frac{C_{0}}{2}\|u\|^{2}+\frac{C_{0}}{2}\|\Delta u\|^{2} .
$$

Thus

$$
\frac{1}{2} \frac{d}{d t}\|u\|^{2}+\left(\gamma-\frac{C_{0}}{2}\right)\|\Delta u\|^{2} \leq \frac{C_{0}}{2}\|u\|^{2} .
$$

In addition, by Poincaré's inequality for functions in $\mathcal{U}_{k}$,

$$
\|u\|^{2} \leq C_{1}\|\nabla u\|^{2}+C_{2},
$$

where $C_{1}$ and $C_{2}$ depend only on $n$ and $\Omega$. We also have

$$
C_{1}\|\nabla u\|^{2} \leq C_{1}\|u\| \cdot\|\Delta u\| \leq \frac{1}{2}\|u\|^{2}+M\|\Delta u\|^{2},
$$

where $M$ is a positive constant. Hence

$$
\|u\|^{2} \leq 2 M\|\Delta u\|^{2}+2 C_{2} .
$$

On the other hand, using (3.3), we finally arrive at

$$
\frac{1}{2} \frac{d}{d t}\|u\|^{2}+\frac{1}{2 M}\left(\gamma-\frac{C_{0}}{2}\right)\|u\|^{2} \leq \frac{C_{0}}{2}\|u\|^{2}+\frac{C_{2}}{M}\left(\gamma-\frac{C_{0}}{2}\right) .
$$


Using (3.4), we obtain

$$
\frac{d}{d t}\|u\|^{2}+\left(\frac{\gamma}{M}-\frac{C_{0}}{2 M}-C_{0}\right)\|u\|^{2} \leq \frac{C_{2}\left(2 \gamma-C_{0}\right)}{M} .
$$

Taking $\gamma$ satisfying $\frac{\gamma}{M}-\frac{C_{0}}{2 M}-C_{0}>0$ gives

$$
\|u\|^{2} \leq e^{-\left(\frac{\gamma}{M}-\frac{C_{0}}{2 M}-C_{0}\right) t}\left\|u_{0}\right\|^{2}+\frac{2 C_{2}\left(2 \gamma-C_{0}\right)}{2 \gamma-C_{0}-2 C_{0} M} .
$$

Thus, for initial data in any bounded set $B \subset \mathscr{U}_{\kappa}$, there is a uniform time $t_{1}(B)$ depending on $B$ such that for $t \geq t_{1}(B)$,

$$
\|u(x, t)\|^{2} \leq \frac{4 C_{2}\left(2 \gamma-C_{0}\right)}{2 \gamma-C_{0}-2 C_{0} M} .
$$

STEP 2: $H^{1}$ norm estimate. Multiplying (1.1) with $\Delta u$ and integrating over $\Omega$, we obtain

$$
\frac{1}{2} \frac{d}{d t}\|\nabla u\|^{2}+\gamma\|\nabla \Delta u\|^{2}=-\int_{\Omega} \Delta A(u) \Delta u d x-\beta \cdot \int_{\Omega} \nabla B(u) \Delta u d x .
$$

Note that

$$
\Delta A(u)=\left(3 \gamma_{2} u^{2}+2 \gamma_{1} u-1\right) \Delta u+\left(6 \gamma_{2} u+2 \gamma_{1}\right)|\nabla u|^{2} .
$$

Hence

$$
\begin{aligned}
\frac{1}{2} \frac{d}{d t}\|\nabla u\|^{2}+\gamma\|\nabla \Delta u\|^{2}+\gamma_{2}\|u \Delta u\|^{2} & \\
= & -\int_{\Omega}\left(2 \gamma_{2} u^{2}+2 \gamma_{1} u-1\right)|\Delta u|^{2} d x-\beta \cdot \int_{\Omega}\left(\frac{1}{4} u^{4}-\frac{1}{2} u^{2}\right) \nabla \Delta u d x \\
& \quad-\int_{\Omega} 2 \gamma_{1}|\nabla u|^{2} \Delta u d x-6 \int_{\Omega} \gamma_{2} u|\nabla u|^{2} \Delta u d x \\
\leq & \gamma_{2} \int_{\Omega} u^{2}|\Delta u|^{2} d x+C \int_{\Omega}|\nabla u|^{4} d x+C \int_{\Omega}|\Delta u|^{2} d x \\
& +\frac{\gamma}{8} \int_{\Omega}|\nabla \Delta u|^{2} d x+C \int_{\Omega}\left(\frac{1}{4} u^{4}+\frac{1}{2} u^{2}\right)^{2} d x .
\end{aligned}
$$

On the other hand, by Nirenberg-Gagliardo's inequality, we have

$$
\|\nabla u\|_{4} \leq C_{1}\|\nabla \Delta u\|^{1 / 3+n / 12}\|u\|^{2 / 3-n / 12}+C_{2}\|u\| \quad(n \leq 2)
$$

and

$$
\|u\|_{8} \leq C_{1}\|\nabla \Delta u\|^{n / 8}\|u\|^{1-n / 8}+C_{2}\|u\| \quad(n \leq 2) .
$$

By (3.8), (3.9) and Young's inequality, we obtain

$$
C\|\nabla u\|_{4}^{4} \leq \frac{\gamma}{8}\|\nabla \Delta u\|^{2}+C_{0}^{\prime}
$$


and

$$
C\|u\|_{8}^{8} \leq C_{1}^{\prime}\|\nabla \Delta u\|^{2}+C_{2}^{\prime} .
$$

Owing to (3.11), we see that

$$
C \int_{\Omega}\left(\frac{1}{4} u^{4}+\frac{1}{2} u^{2}\right) d x \leq \frac{\gamma}{8}\|\nabla \Delta u\|^{2}+C_{3} .
$$

Using (3.5), we have

$$
\begin{aligned}
\int_{\Omega}|\nabla u|^{2} d x & =-\int_{\Omega} u \cdot \Delta u d x \\
& \leq\left(\int_{\Omega} u^{2} d x\right)^{1 / 2}\left(\int_{\Omega}|\Delta u|^{2} d x\right)^{1 / 2} \leq C\left(\int_{\Omega}|\Delta u|^{2} d x\right)^{1 / 2},
\end{aligned}
$$

and we also have

$$
\begin{aligned}
\int_{\Omega}|\Delta u|^{2} d x & =-\int_{\Omega} \nabla u \cdot \nabla \Delta u d x \\
& \leq\left(\int_{\Omega}|\nabla u|^{2} d x\right)^{1 / 2}\left(\int_{\Omega}|\nabla \Delta u|^{2} d x\right)^{1 / 2} .
\end{aligned}
$$

Hence

$$
C \int_{\Omega}|\Delta u|^{2} d x \leq C\left(\int_{\Omega}|\nabla \Delta u|^{2} d x\right)^{2 / 3} \leq \frac{\gamma}{8}\|\nabla \Delta u\|^{2}+C_{4} .
$$

Adding (3.7), (3.10), (3.12) and (3.15) together gives

$$
\frac{1}{2} \frac{d}{d t}\|\nabla u\|^{2}+\frac{\gamma}{2}\|\nabla \Delta u\|^{2} \leq C_{0}^{\prime}+C_{3}+C_{4} .
$$

On the other hand, by (3.13) and (3.14), we obtain

$$
\|\nabla u\|^{2} \leq C\|\nabla \Delta u\|^{2 / 3} \leq C^{\prime \prime}\|\nabla \Delta u\|^{2}+C^{\prime} .
$$

By the above inequality and using (3.16), we have

$$
\frac{d}{d t}\|\nabla u\|^{2}+\frac{\gamma}{C^{\prime \prime}}\|\nabla u\|^{2} \leq 2\left(C_{0}^{\prime}+C_{3}+C_{4}\right)+\frac{\gamma C^{\prime}}{C^{\prime \prime}},
$$

which gives

$$
\|\nabla u\|^{2} \leq e^{-\frac{\gamma}{C^{\prime \prime}} t}\left\|\nabla u_{0}\right\|^{2}+\frac{2 C^{\prime \prime}}{\gamma}\left(C_{0}^{\prime}+C_{3}+C_{4}\right)+C^{\prime} .
$$

Thus for initial data in any bounded set $B \subset \mathscr{U}_{\kappa}$, there is a uniform time $t_{2}(B)$ depending on $B$ such that for $t \geq t_{2}(B)$,

$$
\|\nabla u\|^{2} \leq \frac{4 C^{\prime \prime}}{\gamma}\left(C_{0}^{\prime}+C_{3}+C_{4}\right)+2 C^{\prime} .
$$

Adding (3.5) and (3.18) together, we have

$$
\|u(t)\|_{H^{1}} \leq C .
$$


By Sobolev's imbedding theorem, we finally arrive at

$$
\|u(t)\|_{6} \leq C \text { and }\|u(t)\|_{12} \leq C .
$$

STEP 3: $H^{2}$ norm estimate. Multiplying (1.1) by $\Delta^{2} u$ and integrating the result over $\Omega$, we obtain

$$
\frac{1}{2} \frac{d}{d t}\|\Delta u\|^{2}+\gamma\left\|\Delta^{2} u\right\|^{2}=\int_{\Omega} \Delta^{2} u \Delta A(u) d x+\beta \cdot \int_{\Omega} \Delta^{2} u \nabla B(u) d x .
$$

Hence

$$
\frac{1}{2} \frac{d}{d t}\|\Delta u\|^{2}+\gamma\left\|\Delta^{2} u\right\|^{2} \leq C_{5}\|\Delta A(u)\|^{2}+C_{6}\|\nabla B(u)\|^{2}+\frac{\gamma}{2}\left\|\Delta^{2} u\right\|^{2} .
$$

Note that

$$
\|\Delta A(u)\|^{2} \leq 2\left(\int_{\Omega}\left|A^{\prime}(u)\right|^{2}|\Delta u|^{2} d x+\int_{\Omega}\left|A^{\prime \prime}(u)\right|^{2}|\nabla u|^{4} d x\right)
$$

$$
\begin{aligned}
& \leq 2\left[\left(\int_{\Omega}\left|A^{\prime}(u)\right|^{3} d x\right)^{2 / 3}\left(\int_{\Omega}|\Delta u|^{6} d x\right)^{1 / 3}+\left(\int_{\Omega}\left|A^{\prime \prime}(u)\right|^{6} d x\right)^{1 / 3}\left(\int_{\Omega}|\nabla u|^{6} d x\right)^{2 / 3}\right] \\
& \leq C_{7}\left[\left(\int_{\Omega}|\Delta u|^{6} d x\right)^{1 / 3}+\left(\int_{\Omega}|\nabla u|^{6} d x\right)^{2 / 3}\right],
\end{aligned}
$$

and

$$
\begin{aligned}
\|\nabla B(u)\|^{2} & \leq \int_{\Omega}\left(u^{3}-u\right)^{2}|\nabla u|^{2} d x \\
& \leq \frac{1}{2} \int_{\Omega}\left(u^{3}-u\right)^{4} d x+\frac{1}{2} \int_{\Omega}|\nabla u|^{4} d x \leq C_{8}+\|\nabla u\|_{4}^{4} .
\end{aligned}
$$

Using Nirenberg-Gagliardo's inequality, we have

$$
\begin{aligned}
& \|\nabla u\|_{4} \leq C_{1}^{\prime}\left\|\Delta^{2} u\right\|^{n / 12}\|\nabla u\|^{1-n / 12}+C_{2}^{\prime}\|\nabla u\| \quad(n \leq 2), \\
& \|\nabla u\|_{6} \leq C_{1}^{\prime}\left\|\Delta^{2} u\right\|^{n / 9}\|\nabla u\|^{1-n / 9}+C_{2}^{\prime}\|\nabla u\| \quad(n \leq 2), \\
& \|\Delta u\|_{6} \leq C_{1}^{\prime}\left\|\Delta^{2} u\right\|^{(3+n) / 9}\|\nabla u\|^{1-(3+n) / 9}+C_{2}^{\prime}\|\nabla u\| \quad(n \leq 2) .
\end{aligned}
$$

Using Young's inequality, we have

$$
\begin{gathered}
\|\nabla u\|_{4}^{4} \leq C_{3}^{\prime}\left\|\Delta^{2} u\right\|^{2 / 3}+C_{4}^{\prime} \leq \varepsilon\left\|\Delta^{2} u\right\|^{2}+C_{\varepsilon}, \\
\|\nabla u\|_{6}^{4} \leq C_{3}^{\prime}\left\|\Delta^{2} u\right\|^{8 / 9}+C_{4}^{\prime} \leq \varepsilon\left\|\Delta^{2} u\right\|^{2}+C_{\varepsilon}, \\
\|\Delta u\|_{6}^{2} \leq C_{3}^{\prime}\left\|\Delta^{2} u\right\|^{10 / 9}+C_{4}^{\prime} \leq \varepsilon\left\|\Delta^{2} u\right\|^{2}+C_{\varepsilon} .
\end{gathered}
$$

Thus, we finally arrive at

$$
\begin{aligned}
\frac{d}{d t}\|\Delta u\|^{2} & +\gamma\left\|\Delta^{2} u\right\|^{2} \\
& \leq 2 C_{5} C_{7}\left(\|\Delta u\|_{L^{6}}^{2}+\|\nabla u\|_{L^{6}}^{4}\right)+2 C_{6}\|\nabla u\|_{4}^{4}+2 C_{6} C_{8} \\
& \leq 4 C_{5} C_{7}\left(\varepsilon\left\|\Delta^{2} u\right\|^{2}+C_{\varepsilon}\right)+2 C_{6} \varepsilon\left\|\Delta^{2} u\right\|^{2}+2 C_{6} C_{\varepsilon}+2 C_{6} C_{8},
\end{aligned}
$$


which means

$$
\frac{d}{d t}\|\Delta u\|^{2}+\left(\gamma-4 C_{5} C_{7} \varepsilon-2 C_{6} \varepsilon\right)\left\|\Delta^{2} u\right\|^{2} \leq C .
$$

Taking $\varepsilon$ small enough, we obtain

$$
\frac{d}{d t}\|\Delta u\|^{2}+C_{9}\left\|\Delta^{2} u\right\|^{2} \leq C,
$$

where $C_{9}>0$ is a constant. By a Calderón-Zygmund type estimate, we have

$$
\frac{d}{d t}\|\Delta u\|^{2}+C^{\prime} C_{9}\left(\|\Delta u\|^{2}+\|\nabla \Delta u\|^{2}\right) \leq C .
$$

By Gronwall's inequality and $\|\Delta u(0)\| \leq R$,

$$
\|\Delta u\|^{2} \leq e^{-C^{\prime} C_{9} t}\left\|\Delta u_{0}\right\|^{2}+\frac{C}{C^{\prime} C_{9}} \leq e^{-C^{\prime} C_{9} t} R^{2}+\frac{C}{C^{\prime} C_{9}} \leq \frac{2 C}{C^{\prime} C_{9}}
$$

for $t \geq t_{3}(B)=\frac{1}{C^{\prime} C_{9}} \ln \frac{C^{\prime} C_{9} R^{2}}{C}$. Adding 3.19 and 3.26 together, we obtain

$$
\|u(t)\|_{H^{2}} \leq C \text {. }
$$

By Sobolev's imbedding theorem, we obtain

$$
\|u(t)\|_{L^{\infty}} \leq C \text {. }
$$

By setting $t_{0}(B)=\max \left\{t_{1}(B), t_{2}(B), t_{3}(B)\right\}$, the lemma is proved.

The above lemma implies that $\{S(t)\}_{t>0}$ has a bounded absorbing set in $\mathscr{U}_{\kappa}$. In what follows we prove the precompactness of the orbit in $\mathscr{U}_{\kappa}$.

LEMma 3.2. For initial data $u_{0}$ varying in a bounded set $B \subset \mathscr{U}_{\kappa}$, there exists a $t_{1}(B)>0$ such that

$$
\|u(t)\|_{H^{3}} \leq C, \quad \forall t \geq t_{1}>0
$$

which shows that $\bigcup_{t \geq t_{1}} u(t)$ is relatively compact in $\mathscr{U}_{\kappa}$.

Proof. The uniform bound of the $H^{2}(\Omega)$ norm of $u(t)$ has been obtained in the above lemma. In what follows we derive an estimate on the $H^{3}$ norm.

Applying $\Delta$ to equation 1.1 , we obtain

$$
\frac{\partial \Delta u}{\partial t}+\gamma \Delta^{3} u=\Delta^{2} A(u)+\beta \cdot \nabla \Delta B(u)
$$

with

$$
\left.\varphi\right|_{x_{i}=0}=\left.\varphi\right|_{x_{i}=L_{i}}, \quad i=1,2,
$$

for $u$ and the derivatives of $u$ at least of order $\geq 2$ and $\leq 5$.

Multiplying 3.28 by $\Delta^{2} u$ and integrating over $\Omega$, we obtain

$$
\begin{aligned}
\frac{1}{2} \frac{d}{d t}\|\nabla \Delta u\|^{2} & +\gamma \int_{\Omega}\left|\nabla \Delta^{2} u\right|^{2} d x \\
& -\int_{\Omega} \nabla \Delta A(u) \nabla \Delta^{2} u d x-\beta \cdot \int_{\Omega} \Delta B(u) \nabla \Delta^{2} u d x=0 .
\end{aligned}
$$


Note that

$$
\nabla \Delta A(u)=\nabla\left(A^{\prime}(u) \Delta u+A^{\prime \prime}(u)|\nabla u|^{2}\right)
$$

and

$$
\Delta B(u)=\nabla\left(B^{\prime}(u) \nabla u\right)
$$

Hence

$$
\begin{aligned}
\mid \int_{\Omega} \nabla \Delta & A(u) \cdot \nabla \Delta^{2} u d x \mid \\
= & \left|\int_{\Omega} \nabla\left(A^{\prime}(u) \Delta u+A^{\prime \prime}(u)|\nabla u|^{2}\right) \nabla \Delta^{2} u d x\right| \\
\leq & \left(\int_{\Omega}\left|A^{\prime}(u) \nabla \Delta u \nabla \Delta^{2} u\right| d x+3 \int_{\Omega}\left|A^{\prime \prime}(u) \nabla u \Delta u \nabla \Delta^{2} u\right| d x\right. \\
& \left.+\int_{\Omega}\left|A^{\prime \prime \prime}(u)(\nabla u)^{3} \nabla \Delta^{2} u\right| d x\right) \\
\leq & C\left(\int_{\Omega}\left|\nabla \Delta u \nabla \Delta^{2} u\right| d x+3 \int_{\Omega}\left|\nabla u \Delta u \nabla \Delta^{2} u\right| d x\right. \\
& \left.+\int_{\Omega}\left|(\nabla u)^{3} \nabla \Delta^{2} u\right| d x\right) \\
\leq & C_{10}\left(\|\nabla \Delta u\|^{2}+\|\nabla u \Delta u\|^{2}+\|\nabla u\|_{6}^{6}\right)+C_{11}\left\|\nabla \Delta^{2} u\right\|^{2}
\end{aligned}
$$

and

$$
\begin{aligned}
\mid \int_{\Omega} \Delta B(u) & \nabla \Delta^{2} u d x|=| \int_{\Omega} \nabla\left(B^{\prime}(u) \nabla u\right) \nabla \Delta^{2} u d x \mid \\
& \leq \int_{\Omega}\left|B^{\prime}(u) \Delta u \nabla \Delta^{2} u\right| d x+\int_{\Omega}\left|B^{\prime \prime}(u)(\nabla u)^{2}\right| \nabla \Delta^{2} u \mid d x \\
& \leq C\left(\int_{\Omega}\left|\Delta u \nabla \Delta^{2} u\right| d x+\int_{\Omega}|\nabla u|^{2}\left|\nabla \Delta^{2} u\right| d x\right) \\
\leq & C_{12}\left(\|\Delta u\|^{2}+\|\nabla u\|_{4}^{4}\right)+C_{13}\left\|\nabla \Delta^{2} u\right\|^{2} .
\end{aligned}
$$

On the other hand, by Nirenberg-Gagliardo's inequality, when $n \leq 2$, we obtain

$$
C_{12}\|\nabla u\|_{4}^{4} \leq C\left(\left\|\nabla \Delta^{2} u\right\|^{n / 4}\|\nabla u\|^{4-n / 4}+\|\nabla u\|^{4}\right) \leq \varepsilon\left\|\nabla \Delta^{2} u\right\|^{2}+C_{\varepsilon},
$$

and we also have

$$
C_{10}\|\nabla u \Delta u\|^{2} \leq C\|\nabla u\|_{L^{\infty}}^{2}\|\Delta u\|^{2} \leq C_{14}\|\nabla u\|_{L^{\infty}}^{2} .
$$

By Nirenberg-Gagliardo's inequality again, we obtain

$$
\begin{aligned}
\|\nabla u\|_{\infty}^{2} & \leq C\left(\left\|\nabla \Delta^{2} u\right\|^{n / 4}\|\nabla u\|^{2-n / 4}+\|\nabla u\|^{2}\right) \\
& \leq \varepsilon\left\|\nabla \Delta^{2} u\right\|^{2}+C_{\varepsilon} \quad(n \leq 2)
\end{aligned}
$$


and

$$
\begin{aligned}
\|\nabla u\|_{6}^{6} & \leq C\left(\left\|\nabla \Delta^{2} u\right\|^{n / 2}\|\nabla u\|^{6-n / 2}+\|\nabla u\|^{6}\right) \\
& \leq \varepsilon\left\|\nabla \Delta^{2} u\right\|^{2}+C_{\varepsilon} \quad(n \leq 2) .
\end{aligned}
$$

Thus using $3.29-3.35$, we obtain

$$
\begin{aligned}
\frac{1}{2} \frac{d}{d t}\|\nabla \Delta u\|^{2}+\left[\gamma-C_{11}-C_{13}|\beta|-\left(C_{10}+C_{14}\right.\right. & +|\beta|) \varepsilon]\left\|\nabla \Delta^{2} u\right\|^{2} \\
& \leq C_{10}\|\nabla \Delta u\|^{2}+C_{15}
\end{aligned}
$$

Taking $\varepsilon$ small enough and $\gamma$ satisfying $\gamma-C_{11}-C_{13}|\beta|-\left(C_{10}+C_{14}+|\beta|\right) \varepsilon$ $>0$, by Young's inequality, we have

$$
\frac{1}{2} \frac{d}{d t}\|\nabla \Delta u\|^{2} \leq C_{10}\|\nabla \Delta u\|^{2}+C_{15} .
$$

On the other hand, integrating (3.25) between $t$ and $t+1$, using (3.26), we have

$$
C^{\prime} C_{9} \int_{t}^{t+1}\|\nabla \Delta u\|^{2} d \tau \leq\|\Delta u(t)\|^{2}+C \leq C\left(\frac{2}{C^{\prime} C_{9}}+1\right) .
$$

Owing to (3.36), 3.37) and the uniform Gronwall inequality in [10, we get

$$
\|\nabla \Delta u\|^{2} \leq C, \quad t \geq 1
$$

The lemma is proved.

Then by Theorem I.1.1 of [10], we immediately conclude that $\mathscr{A}_{\kappa}=$ $\omega(\mathscr{B})$. The $\omega$-limit set of the absorbing set $\mathscr{B}$ is a global attractor in $\mathscr{U}_{\kappa}$. By Lemma 3.2, this global attractor is a bounded set in $H^{3}(\Omega)$. Thus the proof of Theorem 2.1 is complete.

\section{References}

[1] J. W. Cholewa and T. Dlotko, Global attractor for the Cahn-Hilliard system, Bull. Austral. Math. Soc. 49 (1994), 277-292.

[2] T. Dlotko, Global attractor for the Cahn-Hilliard equation in $H^{2}$ and $H^{3}$, J. Differential Equations 113 (1994), 381-393.

[3] A. Eden and V. K. Kalantarov, The convective Cahn-Hilliard equation, Appl. Math. Lett. 20 (2007), 455-461.

[4] -, - 3D convective Cahn-Hilliard equation, Comm. Pure Appl. Anal. 6 (2007), $1075-1086$.

[5] A. A. Golovin, S. H. Davis and A. A. Nepomnyashchy, A convective Cahn-Hilliard model for the formation of facets and corners in crystal growth, Phys. D 122 (1998), $202-230$.

[6] K. H. Kwek, On the Cahn-Hilliard type equation, $\mathrm{PhD}$ thesis, Georgia Institute of Technology, 1991.

[7] D. S. Li and C. K. Zhong, Global attractor for the Cahn-Hilliard system with fast growing nonlinearity, J. Differential Equations 149 (1998), 191-210. 
[8] C. C. Liu, On the convective Cahn-Hilliard equation, Bull. Polish Acad. Sci. Math. 53 (2005), 299-314.

[9] —, On the convective Cahn-Hilliard equation with degenerate mobility, J. Math. Anal. Appl. 344 (2008), 124-144.

[10] R. Temam, Infinite-Dimensional Dynamical Systems in Mechanics and Physics, Springer, New York, 1988.

[11] S. J. Watson, F. Otto, B. Y. Rubinstein and S. H. Davis, Coarsening dynamics of the convective Cahn-Hilliard equations, Phys. D 178 (2003), 127-148.

[12] H. Wu and S. M. Zheng, Global attractor for the 1-D thin film equation, Asymptotic Anal. 51 (2007), 101-111.

[13] M. A. Zaks, A. Podolny, A. A. Nepomnyashchy and A. A. Golovin, Periodic stationary patterns governed by a convective Cahn-Hilliard equation, SIAM J. Appl. Math. 66 (2005), 700-720.

Xiaopeng Zhao, Changchun Liu

Department of Mathematics

Jilin University

Changchun 130012, P.R. China

E-mail: zxp032@126.com

Received March 6, 2010;

received in final form July 7, 2010 